\title{
Constraints on spacing between transcription factor binding sites in a simple adenovirus promoter
}

\author{
Lily Wu and Arnold Berk \\ Molecular Biology Institute and Department of Microbiology, University of California at Los Angeles, Los Angeles, California \\ 90024 USA
}

\begin{abstract}
The adenovirus 2 E1B transcription unit has an extremely simple promoter consisting of a TATA box and a closely situated GC box. The interaction between the TATA box and the GC box was analyzed using insertion mutations which expand the distance between the two elements. We observed that the E1B promoter has an unusually rigid architecture. When the GC box, which is a binding site for transcription factor Sp1, was separated further from the TATA box than in the wild-type promoter, in vivo transcription quickly diminished to a level comparable to the elimination of the Sp1 site. Yet all the insertion mutants bound Sp1 factor in vitro with an affinity approximately equal to that of the wild-type promoter. From these results, we argue that Sp1 binding alone is not sufficient to stimulate transcription. The increased distance might disrupt direct contacts between Sp1 and transcription factors bound at the TATA box, contacts required for transcription stimulation by $S p 1$. The insertion mutations do not interfere with the transcription activation process mediated by the adenovirus large E1A protein or the pseudorabies virus immediate early protein.
\end{abstract}

[Key Words: Adenovirus 2; E1B promoter; promoter mutations; Spl]

Received December 8, 1987; revised version accepted February 16, 1988.

Transcription control signals of eukaryotic genes transcribed by RNA polymerase II are composed of distinct sequence elements usually located upstream of the transcription start site (for reviews, see McKnight and Tjian 1986; Maniatis et al. 1987). The first prominent promoter element is the commonly found TATA box, which is located approximately $30 \mathrm{bp}$ upstream of the transcription start site. Ample evidence indicates that the TATA box plays a fundamental role in transcription initiation (Breathnatch and Chambon 1981). In many promoters, mutations in the TATA box cause a greater decrease in transcription than mutations in other promoter elements do. This is the case for the herpes simplex virus type-I thymidine kinase (HSV tk) (Coen et al. 1986), mouse $\beta$-globin (Charnay et al. 1985), adenovirus 2 (Ad2) E1B (Wu et al. 1987) and major late promoters (Corden et al. 1980; Hu and Manley 1981; Concino et al. 1983). An RNA polymerase II transcription factor has been shown to bind to the TATA box region (Parker and Topol 1984: Sawadogo and Roeder 1985). The second class of promoter signals are the so-called upstream elements that appear to be binding sites for transcription factors. One particularly well-studied example is a GCrich element found in many cellular and viral promoters (Kadonaga et al. 1986). The cellular transcription factor, $\mathrm{Sp} 1$, which binds to this element has been purified to homogeneity (Briggs et al. 1986; Kadonaga and Tiian 1986). Addition of Spl to in vitro transcription reactions stimulates transcription from promoters containing Sp1 binding sites (Dynan and Tjian 1983a,b; Jones et al. 1985). However, the mechanism by which Sp1 and other upstream transcription factors act to modulate transcription initiation remains unclear.

Two modes of upstream transcription factor interaction seem very likely to be crucial to transcription stimulation. The first, a protein-DNA interaction, requires that the transcription factor recognizes and binds to its cognate DNA sequence. In two well-studied systems, the simian virus 40 (SV40) early and HSV tk promoters, DNA sequence mutations that depress RNA synthesis also inhibit transcription factors from binding (Jones et al. 1985; Wildeman et al. 1986; Lee at al. 1987a,b; Mitchell et al. 1987). Second, both biochemical and in vivo genetic analyses suggest that protein-protein interactions between transcription factors are important to the transcription process. Biochemical studies indicate that binding of the upstream factor MLTF (Chodosh et al. 1986) to the adenovirus major late promoter is greatly stabilized by binding of the adjacent TATA-box transcription factor (TFIID) (Sawadogo and Roeder 1985). This observation supports the hypothesis that TFIID and MLTF make protein-protein contacts with each other when they are bound concurrently (Sawadogo and Roeder 1985).

Expansion of the distance between upstream elements and the TATA box can reduce transcription (McKnight 1982; Takahashi et al. 1986). In addition, in the SV40 promoter/enhancer early region, inserting half-turns of a 
DNA helix between transcription factor binding sites is more detrimental to transcription than inserting full turns (Takahashi et al. 1986). These results all point toward the involvement of direct functional interactions between transcription factors. More specifically, the latter result suggests that proper transcription initiation requires stereospecific alignment between upstream factor(s) and the protein(s) bound to the TATA box.

In light of the indications that transcription factors need to interact to augment transcription, it might seem surprising that considerable changes in the distance between the TATA box and the upstream elements can be tolerated (McKnight 1982). Thus, to accommodate the necessary interactions, the plasticity in the allowed positions of promoter elements must stem from either flexibility in the intervening DNA (Ptashne 1986) or in the transcription factor proteins (Takahashi et al. 1986), or both.

In an effort to study the interactions between transcription factors, we have analyzed the influence of alterations in the spacing between transcription factor binding sites in the unusually simple Ad2 E1B promoter. The principle E1B promoter elements are a binding site for the Spl transcription factor (Kadonaga et al. 1987) and a consensus TATA box (Fig. 1; Wu et al. 1987; also see data below). Disruption of the $\mathrm{Spl}$ site and TATA box reduced the rate of E1B transcription in vivo by 6and 20 -fold, respectively. Deletion of both elements eliminated detectable E1B transcription. Transcription from the ElB promoter is greatly stimulated by the Ad2 large E1A protein (reviewed in Berk 1986) and the pseudorabies virus immediate early protein (IEP), a process termed trans-activation. Here, we also analyzed the influence of altered spacing between the E1B promoter elements on ElA and IEP trans-activation.

We found that the distance between the Spl and TATA-box transcription factor binding sites required for maximal promoter activity is unusually constrained in the E1B promoter. This result suggests that direct interactions between these two factors are required for transcription stimulation by $S p 1$. All insertion mutants were activated well by E1A and IEP. This result indicates that disruption of the interaction between Spl and TATAbox transcription factors does not interfere with the transactivation process.

\section{E1B PROMOTER MUTANTS}

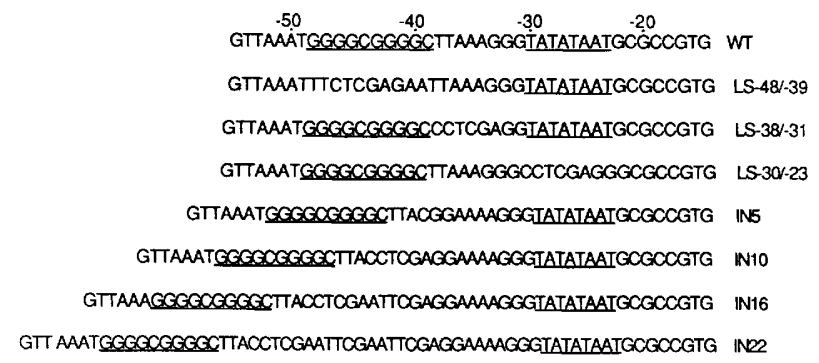

Figure 1. Sequence of the adenovirus $2 \mathrm{E} 1 \mathrm{~B}$ promoter region and mutants. The GC box and TATA box are underlined in the wild-type and mutant promoters.

\section{Results}

No significant E1B promoter elements lie between the Sp1 site and TATA box

An earlier study (Wu et al. 1987) suggested that the Ad2 E1B promoter was comprised principally of single, closely situated binding sites for host cell transcription factors Spl and a TATA-box transcription factor. The high-affinity Spl-binding site GGGGCGGGGC (Kadonaga et al. 1986) occurs between 48 and 39 bp upstream of the Ad2 E1B RNA cap site (Fig. 1) and is bound by purified Spl in vitro (Wu et al. 1987). Deletion of Ad2 sequence from within the neighboring E1A transcription unit to just upstream of this sequence (from -127 to -55 with respect to the E1B cap site) does not depress ElB transcription significantly, whereas deletions and linker-scanning mutations that impinge on the GGGGCGGGGC sequence depress E1B transcription to $15-20 \%$ the level of the wild-type promoter (Wu et al. 1987). However, none of the mutants analyzed previously tested the function of the 8 -bp sequence between the TATA box and the consensus Spl-binding site (Fig. 1). To determine whether this 8-bp sequence con-

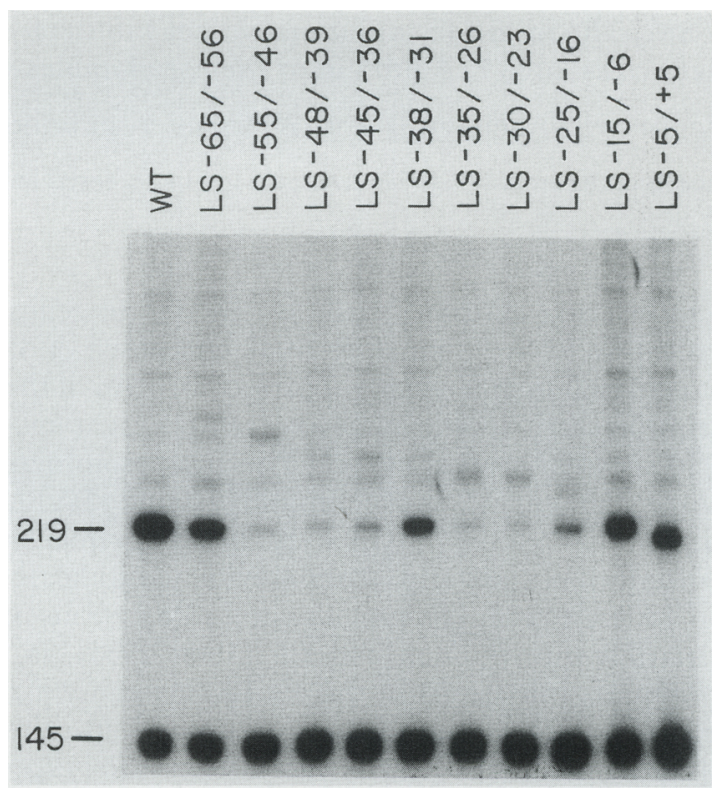

Figure 2. Transcription from E1B promoter linker-scanning mutants. An autoradiogram shows an S1 analysis of RNA isolated $16 \mathrm{hr}$ post-infection of HeLa cells. Cells were coinfected with the mutant indicated at the top of each lane plus the control S13B mutant containing a wild-type E1B promoter and a small deletion at +74 in the E1B transcription unit. Correctly initiated E1B transcripts from the E1B promoter mutant viruses and wild-type protect a 219-base fragment from Sl digestion. E1B transcripts from the internal control S13B mutant produce a 145-base protected fragment. The S1-protected fragment generated from LS $-5 /+5$ is slightly shorter than for the other mutants because of the region of nonhomology between the first 5 bases of the mRNA and the probe generated from wildtype Ad2 DNA. S1 analyses performed with the homologous probe prepared from LS $-5 /+5$ show that the $5^{\prime}$ end of LS $-5 /+5$ E1B RNA maps at the +1 position /data not shown). 
tained a significant promoter element, we constructed the mutant LS - 38/ - 31 in which this region is substituted with an unrelated DNA sequence (Fig. 1). We also constructed mutant LS $-30 /-23$, which has a precise substitution of the TATA box, and mutant LS - 48/ - 39, which has a precise substitution of the consensus $\mathrm{Spl}$-binding site.

Figure 2 shows an S1 analysis of E1B RNA isolated 16 hr postinfection of HeLa cells with LS $-48 /-39$, LS $-38 /-31$, and LS $-30 /-23$, as well as the E1B promoter LS mutants constructed earlier (Wu et al. 1987). To provide an internal control for transcription from the wild-type E1B promoter, the cells were coinfected with the mutant S13B, which has a small deletion at +74 in the E1B transcription unit. Correctly initiated E1B transcripts from the E1B-promoter mutant viruses protect a 219-base fragment from S1 digestion, whereas ElB transcripts from the S13B virus produce a 145-base protected fragment. All mutations disrupting the GGGGCGGGGC Sp1-binding site consensus sequence (LS - 55/ - 46, LS - 48/ - 38, and LS - 45/ - 36) or the TATA box (LS $-35 /-26$, LS $-30 /-23$, and LS - 25/ - 16) depressed E1B transcription significantly. However, mutations that did not disrupt the Spl consensus sequence or the TATA box, including LS - 38/ - 31, had only minor effects on E1B transcription (Fig. 2). The level of transcription from each of these mutants relative to the wild-type E1B promoter was similar when RNA was isolated during the early phase of infection ( $6 \mathrm{hr}$ postinfection; data not shown).

These results demonstrate that sequences in the interval between the Spl consensus sequence and the
TATA box have only a minor influence on E1B transcription. However, a slight depression in E1B transcription from mutant LS - 38/ - 31, compared to the wildtype promoter, was reproducibly observed. This effect was probably due to a slight decrease in the affinity of Spl for the LS - 38/ - 31 template, compared to the wild-type promoter. Figure 3 shows a titration of the DNA-binding activity of purified Spl on the wild-type E1B promoter and LS - 38/ - 31, as assayed by DNase I footprinting. In this and other experiments, concentrations of $\mathrm{Sp} 1$ four to eight times higher were required to completely protect an approximately 20 bp region centered over the GGGGCGGGGC sequence in LS - 38/ - 31, compared to the wild-type promoter. As expected, no protection by $\mathrm{Spl}$ was observed for the mutant LS - 48/ - 39. A similar decrease of four- to eightfold in the affinity of Spl for the LS - 38/ - 31 mutant was observed in competition assays where unlabeled DNA fragments were used to compete for $\mathrm{Sp} 1$ binding to a labeled wild-type fragment (as in Fig. 4 below; data not shown). These data indicate that nucleotides just $3^{\prime}$ to the 10-bp consensus sequence have a small influence on Spl binding. These results, together with those presented previously (Wu et al. 1987), demonstrate that the principle E1B promoter elements are the single Sp1binding site and the closely situated TATA box.

Mutants with expanded distances between the Sp1 site and TATA box

Insertion mutations in the E1B promoter region were generated by linker insertion (Barany 1985), as described

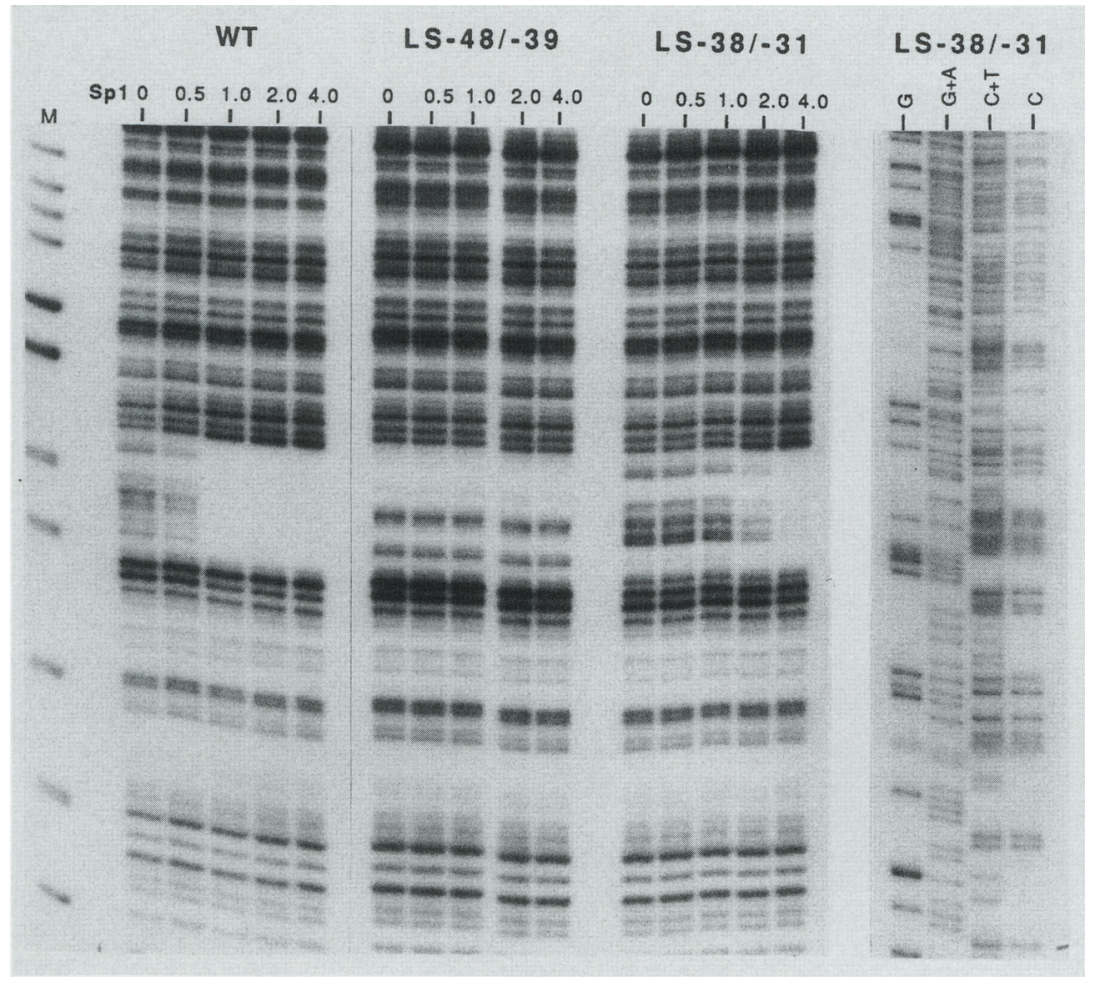

Figure 3. Spl DNase I footprinting on wildtype and ElB promoter mutants. The DNA probes used in the footprinting analyses are indicated at the top. Probes of equal specific activity were incubated in binding reactions with microliters of purified Spl indicated at the top of each lane and then digested with DNase $I$, as described in Materials and methods. (M) A lane of markers. Maxam-Gilbert sequencing of the LS $-38 /-31$ probe is shown in the four lanes at the right. 


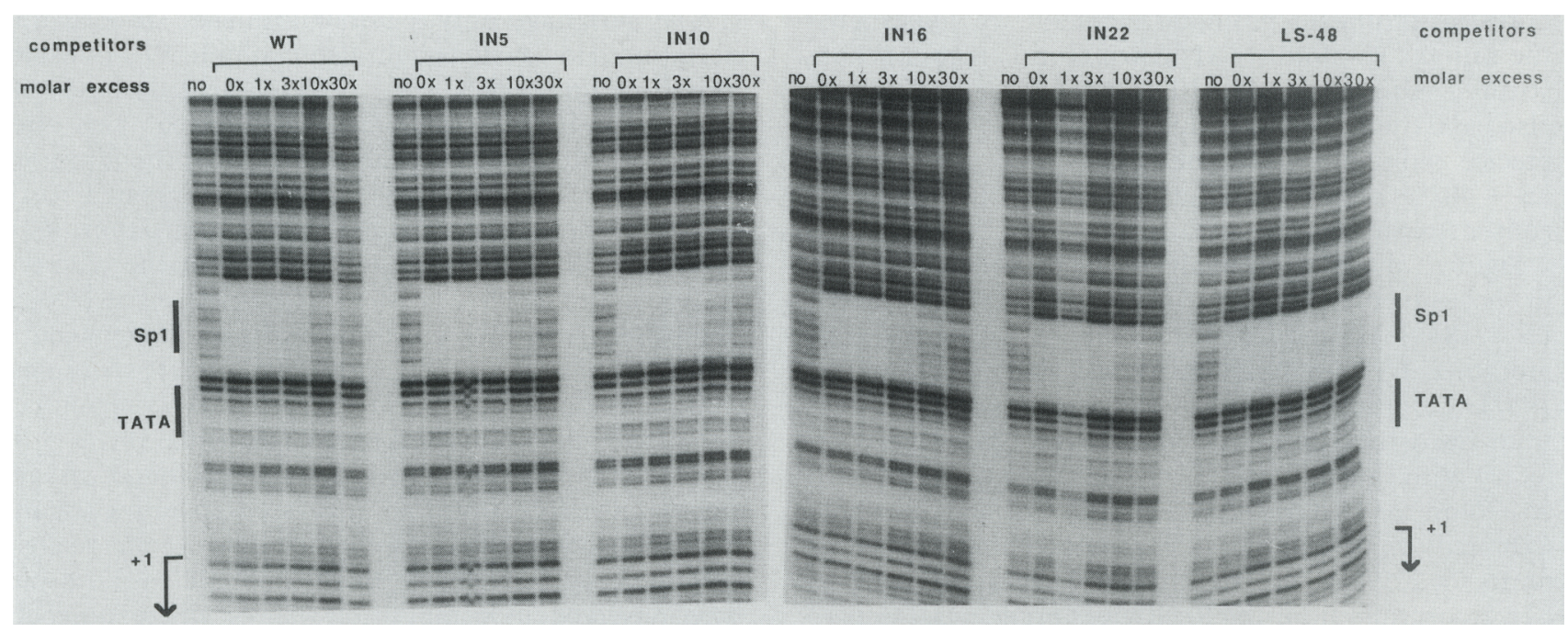

Figure 4. DNase I footprinting competition by the ElB promoter insertion mutants. DNase I footprinting shown in each panel was performed using the wild-type promoter region as probe and partially purified Spl, as described in Materials and methods. (Lanes no) Reactions without added Spl. All other lanes contained the same amount of Spl and the indicated molar excess over the labeled probe of the equivalent restriction fragments from the wild-type or mutant promoter regions, as indicated. The unlabeled, competing fragments extend from -362 to +74 .

in Materials and methods. The precise sequence alterations in these mutants were determined by DNA sequencing (Fig. 1). The insertion mutations were designed to merely expand the distance between, while maintaining the integrity of, the $\mathrm{Spl}$ consensus (Kadonaga et al. 1986) and TATA-box sequences. Furthermore, the insertions are multiples of approximately half-turns of the DNA helix, allowing us to investigate the stereospecificity (Takahashi et al. 1986) of Spl function. The Spl affinity and the transcriptional activities of these insertion mutants were compared with the wild-type promoter and the LS - 48/ - 39 mutant whose Spl site was substituted precisely. To facilitate the accurate analyses of E1B transcription in vivo, we chose to rebuild all mutants into the adenoviral genome. In this way, the ElB promoter mutations can be introduced into HeLa cells by viral infection. These mutant viruses were also designed to contain a deletion in their E1A gene (dl1505; Osborne et al. 1984), which rendered them deficient in ElA transactivation function. ElA activation of the mutants was examined by coinfection with adenovirus mutant S13B, which provides wild-type E1A function in trans but expresses a distinguishable E1B message. Likewise, transactivation by IEP of pseudorabies virus was examined by coinfection with wild-type pseudorabies virus.

\section{Binding of Sp1 to the promoter mutants}

The first point to establish regarding the effects of the insertions was whether the Spl transcription factor recognizes and binds to the mutated promoters with an affinity equal to that of the wild-type promoter. The Sp1binding affinity to different mutants was compared by a DNase I footprinting competition assay, as shown in Figure 4. Using a wild-type E1B promoter fragment as probe, partially purified Spl (Briggs et al. 1986) clearly protected a DNA segment containing the GGGGCGGGGC element from DNAse I cleavage. The experiments also show the titration of excess unlabeled wild-type or mutant competitor promoter fragment. When 10- to 30-fold molar excess of wild-type fragment was added, the Spl-binding was entirely competed away. Notably, the insertion mutants IN5, IN10, IN16, and IN22 competed as efficiently as the wild-type E1B promoter because the same titration endpoint was reached with 10- to 30-fold molar excess of the mutant promoter fragments. We also observed in direct DNAse I footprinting analyses of each of the labeled mutant promoters that the partially purified Spl bound to the wildtype and insertion mutant promoters at equal Spl concentrations (data not shown). These results indicate that $\mathrm{Spl}$ has similar affinity (within a factor of 2) for each of the insertion mutant promoters as for the wild-type sequence. On the other hand, the GC-box substitution mutant LS - 48/ - 39 did not compete (Fig. 4) and did not bind $\mathrm{Sp} 1$ in direct footprinting experiments (Fig. 3), as expected.

\section{E1B transcription of the insertion mutants}

The DNAse I footprinting results clearly show that Spl can recognize and interact with the insertion mutants. To determine what effect the increased distance between the Spl site and TATA box has on E1B transcription, HeLa cells were infected with mutant viruses and cytoplasmic RNA was harvested after $6 \mathrm{hr}$, during the early phase of infection. E1B RNA concentrations were examined either without expression of transactivator protein or in the presence of E1A or IEP. E1B RNA was quantitated by S1 analysis. Much less E1B RNA was expressed from IN16 and IN22 than from the wild-type E1B promoter (Fig. 5; Table 1). This was true both for the 
unstimulated promoter and under conditions of transactivation by E1A or IEP (Fig. 5; Table 1). In fact, E1B RNA from these mutants was reduced to a similar level as observed for the Spl-site substitution mutant LS - 48/

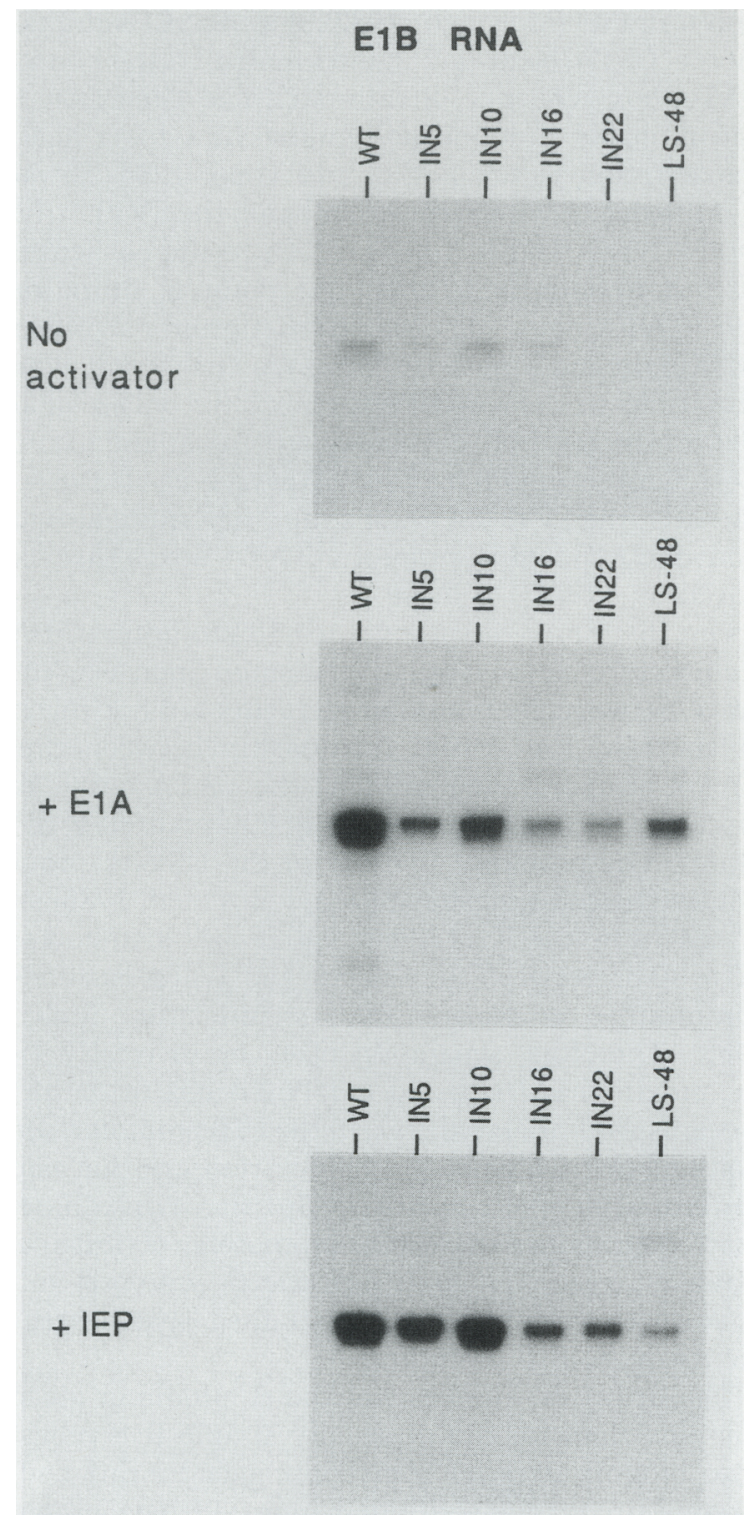

Figure 5. Transcription from the wild-type and E1B promoter insertion mutants. S1 analyses of cytoplasmic RNA isolated 6 $\mathrm{hr}$ postinfection were performed as described in Materials and methods. In the +E1A panel, cells were coinfected with Ad5 mutant S13B, which provided wild-type ElA proteins in trans. In the + IEP panel, cells were coinfected with pseudorabies virus to provide IEP in trans. The infections were performed at the same time, the S1 analyses were performed with the same probe, and the autoradiograms were exposed for the same period. Consequently, the intensities of the bands represent the relative concentrations of E1B mRNAs. Quantitation of this data is shown in Table 1, experiment 1 . The reproduction of the upper panel shown here appears to show the intensity of the IN5 S1-protected fragment equal to that of the IN16 mutant, but this is an artifact of the reproduction process. The correct quantitations obtained from longer exposures of the original autoradiogram are listed in Table 1.
Table 1. Transcriptional activity of the E1B mutants relative to wild type (WT)

\begin{tabular}{lllllll}
\hline & & \multicolumn{4}{c}{ Experiment } & \\
\cline { 3 - 6 } & & 1 & 2 & 3 & 4 & Mean \pm S.D. \\
\hline \multirow{4}{*}{ No } & WT & 1.00 & 1.00 & 1.00 & 1.00 & 1.00 \\
activator & IN5 & 0.71 & 1.06 & 0.67 & 0.97 & $0.85 \pm 0.17$ \\
& IN10 & 1.55 & 1.00 & 0.64 & 1.09 & $1.07 \pm 0.32$ \\
& IN16 & 0.18 & 0.22 & 0.17 & 0.31 & $0.22 \pm 0.055$ \\
& IN22 & 0.15 & 0.08 & 0.13 & 0.11 & $0.12 \pm 0.025$ \\
& LS - 48 & 0.18 & 0.14 & 0.13 & 0.27 & $0.18 \pm 0.055$ \\
& WT & 1.00 & 1.00 & 1.00 & 1.00 & 1.00 \\
+ E1A & IN5 & 0.38 & 1.00 & 0.32 & 0.38 & $0.52 \pm 0.28$ \\
& IN10 & 0.47 & 1.22 & 0.48 & 0.34 & $0.63 \pm 0.35$ \\
& IN16 & 0.11 & 0.19 & 0.07 & 0.08 & $0.11 \pm 0.047$ \\
& IN22 & 0.11 & 0.17 & 0.06 & 0.05 & $0.10 \pm 0.048$ \\
& LS - 48 & 0.26 & 0.17 & 0.06 & 0.23 & $0.18 \pm 0.082$ \\
& WT & 1.00 & 1.00 & & & 1.00 \\
+ IEP & IN5 & 0.40 & 0.98 & & & 0.69 \\
& IN10 & 1.00 & 1.19 & & & 1.09 \\
& IN16 & 0.27 & 0.31 & & & 0.29 \\
& IN22 & 0.34 & 0.18 & & & 0.26 \\
& LS - 48 & 0.20 & 0.10 & & & 0.15 \\
\hline
\end{tabular}

-39 ( $\sim 0.18$ of wild-type, Table 1). Insertion of 5 or $10 \mathrm{bp}$ was better tolerated. E1B RNA produced by IN10 was not reduced significantly compared to wild-type (Table I). In the experiment shown in Figure 5 (Experiment 1 of Table 1), IN5 produced less E1B RNA than IN10 or wildtype did. However, this phasic variation was not observed in all experiments (Table 1). Finally, the insertions did not alter the start site of E1B transcription (Fig. 5).

\section{Transcription activation by E1A and IEP}

To determine whether the insertions interfere with transactivation, the influence on E1B transcription of ElA and IEP expression was compared between the wild-type and mutant promoters (Fig. 5). E2A RNA expression was examined in each experiment as a control for recovery of mRNA and for the function of E1A and IEP transactivators. Equivalent levels of E2A RNA were detected in each of the coinfections with S13B (to provide EIA) and pseudorabies virus (to provide IEP) (data not shown). In the absence of a viral transactivator, insufficient E2A RNA was produced to be detected under the conditions used $1 \leqslant 2 \%$ the level of E2A RNA observed in the S13B coinfection). The E1B RNA produced from the wild-type promoter was activated about 10-fold by E1A. All mutants were activated similarly by E1A, ranging from 7 - to 14 -fold. Similarly, the pseudorabies virus IEP stimulated the mutant promoters, as well as the wild-type E1B promoter (Fig. 5). Therefore, the insertions do not interrupt transactivation mediated by either E1A or IEP.

\section{Discussion}

To understand the functional relationship between the 
Spl site and TATA box of the E1B promoter, we investigated the effects of expanding the distance between the two sites. We observed that when the Sp1 site was positioned farther than 18 bp from the TATA box, its effect on transcription diminished significantly. However, we showed that the affinity of Spl for the displaced binding sites in each of the insertion mutants was unaltered. In contrast, the LS - 48/ - 39 mutant, whose Spl site was substituted precisely, could not bind $\mathrm{Spl}$. In vivo transcription from this E1B promoter mutant fell to $18 \pm 5.5 \%$ of wild-type during the early phase of infection. As observed for other promoter regions containing Spl sites, such as the SV40 early (Dynan and Tjian 1983b) and HSV tk (Jones et al. 1985) promoters, we conclude that for the $\mathrm{Spl}$ transcription factor to participate in the transcription initiation process, it must first recognize and bind to the promoter region. However, Sp1 binding to the promoter region alone is not sufficient for its function. In the simple E1B promoter, the influence of $\mathrm{Spl}$ fell off rapidly, comparing a promoter with $18 \mathrm{bp}$ between the Spl site and TATA box and promoters with 24 and $30 \mathrm{bp}$ separating these transcription factor binding sites.

A simple interpretation could be that besides binding to the DNA, the $\mathrm{Sp} 1$ factor must also contact the TATA box factor directly to mediate its influence on transcription. The added distance in IN16 and IN22 might prevent such contact between the two transcription factors. Consequently, the transcription phenotype of IN16 and IN22 is depressed comparably to the Sp1 site-deficient mutant, LS - 48/ - 39. Interestingly, the IN5 and IN10 mutations cause a much smaller depression in transcription. Perhaps these smaller insertions are better tolerated because of flexibility in the transcription factor proteins. For example, a flexible protein hinge might separate a DNA-binding domain from a domain that contacts a neighboring transcription factor, as suggested by Takahashi et al. (1986).

Inserting a half-turn of DNA helix between the Sp1 site and TATA box (IN5) was more detrimental to E1B transcription than insertion of a full turn (IN10) in some experiments (Fig. 5), as might be expected even for 'flexible' transcription factors. However, the effect was somewhat smaller and was observed less consistently (Table 1) than for similar insertions between transcription factor binding sites in the SV40 early promoter (Takahashi et al. 1986). Perhaps this is because multiple Spl factors bind along one side of the helix in the SV40 21-bp repeat region (Gidoni et al. 1984). The additive effects of these multiple, assymetrically arranged transcription factors might increase the stereospecificity of their interactions with the TATA-box transcription factor or enhancer-binding factors (Wildeman et al. 1986; Lee et al. 1987b; Mitchell et al. 1987).

In the early SV40 promoter, Takahashi et al. (1986) observed that insertions between the Spl sites and the TATA box result in new transcription start sites. These are displaced upstream by a distance approximately equal to the size of the insertion, so that it appears that the $\mathrm{Sp} 1$ binding sites are more important than the TATA box in positioning the transcription start sites in the early SV40 promoter. In the E1B promoter insertion mutants studied here, no displacement in the transcription start site was observed. Nor is there a change in the transcription initiation site in HSV I tk promoter insertion mutants (McKnight 1982). Both the E1B and tk promoter TATA boxes are a good match to the consensus TATA-box sequence (Breathnatch and Chambon 1981) and probably function as binding sites for a host cell transcription factor (Parker and Topol 1984; Sawadogo and Roeder 1985). On the other hand, the SV40 early promoter TATA box is a poor match to the consensus sequence. In both the E1B and tk promoters, mutations in the TATA box depress transcription significantly (McKnight and Kingsbury 1982; Wu et al. 1987), whereas mutations in the SV40 TATA box have only a small effect on the level of transcription (Benoist and Chambon 1981; Fromm and Berg 1983; Wasylyk et al. 1983). Apparently, the SV40 TATA box functions quite differently from the E1B and tk promoter TATA boxes that fix the initiation site.

One distinctive feature of the E1B promoter is its rigid architecture, in that relatively small increases in distance between its promoter elements are not tolerated. In contrast, the spatial relationship between the upstream promoter elements and the TATA box is much more flexible in the extensively analyzed HSV tk promoter. The proximal Sp1 site in the HSV tk promoter (McKnight and Kingsbury 1982; Jones et al. 1985) is separated from the TATA box by $19 \mathrm{bp}$. Introducing an extra 30 bp between the Spl site and TATA box does not compromise tk transcription significantly (McKnight 1982). Therefore, separation of $49 \mathrm{bp}$ in total between the proximal Spl site and the TATA box in the tk promoter is well tolerated. Yet, in the E1B promoter, separations of just 24 or $30 \mathrm{bp}$ reduce transcription equivalently to a complete loss of the Sp1 site. What characteristics distinguish these two promoters, permitting the extensive spatial flexibility of the tk promoter in contrast to the much more constrained spacing requirements in the E1B promoter?

The upstream elements of the tk promoter are more complex than for the unusually simple ElB promoter. Two binding sites for transcription factor Spl and one binding site for transcription factor CTF have been identified (Jones et al. 1985) in the upstream control elements defined and studied by McKnight and co-workers (McKnight and Kingsbury 1982; McKnight 1982). Transcription factor CTF binds to a CCAAT box at -81 to -85 , which is flanked by two $\mathrm{Spl}$ sites (Jones et al. 1985). Perhaps multiple interactions between the upstream transcription factors and the TATA box factor bound to the tk promoter provide sufficient stability to permit deformation and bending of the intervening DNA helix, a mechanism suggested for the long-distance influence of enhancer regions (Ptsahne 1986). Interactions between the single Spl factor and the TATA-box factor bound on the E1B promoter might not be sufficient to permit bending of the intervening DNA. Consistent with this idea, insertion of multiples of a single tran- 
scription factor binding site often stimulate transcription in vivo to a much greater extent than insertion of a single site (Angel et al. 1987; Lee et al. 1987b). Alternatively, the CTF factor bound to the tk promoter region might mediate the influence of the neighboring $\mathrm{Sp} 1$ factors, allowing $\mathrm{Spl}$ interactions over a longer distance.

Transcription from the E1B promoter is stimulated about 10-fold by the large adenovirus E1A protein (Berk et al. 1979; Jones and Shenk 1979a; Nevins 1981) and by the pseudorabies virus IEP (Fig. 5, Feldman et al. 1982). The mechanism of this transactivation is not yet well understood. If these transactivating viral proteins functioned to increase the stimulating effects of upstream factors or to influence interactions between upstream factors and the TATA-box factor, we might have expected that the IN16 and IN22 insertions that nearly eliminate the influence of the EIB Spl site would show diminished transactivation compared to the wild-type promoter. This was not observed. On the other hand, the results are in agreement with the suggestion, based on studies of E1B promoter linker-scan mutants (Wu et al. 1987) and analyses of transcription factor activities in extracts of adenovirus-infected HeLa cells (Leong et al. 1988), that E1A expression increases transcription from the E1B promoter by stimulating the activity of the TATA-box transcription factor. Many of the hypotheses raised by these studies can be tested directly through further manipulations of the E1B promoter sequence and analyses of transcription factor interactions in vitro.

\section{Materials and methods}

\section{Cells and viruses}

Suspension cultures of HeLa and 293 cells (Graham et al. 1977) were grown in minimal essential medium (SMEM, GIBCO) supplemented with 5\% newborn calf serum (NCS). The 293 cells were also grown as monolayers in Dulbecco's modified Eagle's medium (DMEM, GIBCO) containing 10\% NCS.

Adenoviruses containing the ElB promoter mutations were propagated in suspension cultures of 293 cells grown in SMEM containing $2 \%$ NCS. Viral stocks harvested from 293 cells were assayed by plaque titration on 293 monolayers in DMEM with $0.7 \%$ agarose, $2 \mathrm{mM}$ L-glutamine, MEM nonessential amino acids (GIBCO), $25 \mathrm{mM} \mathrm{MgCl}_{2}$, and $2 \%$ NCS. Wild-type pseudorabies virus was obtained from $\mathrm{L}$. Feldman, propagated on rabbit skin cells, and assayed by plaque titration on rabbit kidney cells.

\section{Mutant plasmid construction}

The pBR322 derived plasmids used in this study were propagated in Escherichia coli HB101, and plasmid DNA was purified by the alkaline-SDS method, as described (Birnboim and Doly 1979). Enzymes for cloning were used according to the manufacturers' recommendations.

The E1B promoter insertion mutant IN 10 (insertion of $10 \mathrm{bp}$ ) was created by combining two previously constructed linker scan mutants, LS $-45 /-36$, LS $-35 /-26$ (Wu et al. 1987). The LS mutants have the specified sequence (e.g., from -45 to -36 upstream of the E1B transcription start site) replaced by an XhoI linker sequence (CCTCGAGGAA). Fusing the small $X b a I-X h o I$ fragment of pBE5 dl1500 LS $-35 /-26$ to the large
XbaI-XhoI fragment of pBE5 dl1500 LS - 45/ - 36 resulted in the 10-bp insertion CCTCGAGGAA at -35 . Insertion at -35 does not disrupt either the GC $(-49$ to -39$)$ or TATA boxes $(-30$ to -23$)$.

IN5, IN16, and IN22 mutants were all generated from the IN10 mutant. IN5 was generated by cutting IN10 at the XhoI site and trimming the protruding single-stranded ends with nuclease $S 1$ (BRL). One clone resulting from the blunt end ligation had five residues removed and was designated IN5. IN16 and IN22 were derived by ligating one or two copies, respectively, of a 6-nucleotide single-stranded linker (Pharmacia) into the XhoI site of IN10. The pTCGAAT linker converts the XhoI protruding ends to an EcoRI site (Barany 1985). The precise sequence of each mutation was determined by the dideoxy-chain termination methods of sequencing (Sanger et al. 1977; Smith 1980). Ml3 GGl LS - 48/ - 39 was created by oligonucleotide-directed mutagenesis, as described previously (Zoller and Smith 1983; Wu et al. 1987). A 40-mer oligonucleotide that precisely substituted the 10 residue GC box with an XhoI site (TTCTCGAGAA) was utilized to prime the mutagenesis. M13 GG1 LS - 38/ - 31 and M13 GG1 LS - 30/ - 23 were constructed similarly using 40-mer mutagenic oligonucleotides with the multiple-base substitutions near the center.

To facilitate the construction of these E1B promoter mutations into the adenoviral genome, the XbaI-SacI fragment of each E1B promoter mutant was cloned between the equivalent sites of pBE1505. This plasmid contains Ad2 sequence (1-3329 nucleotide) from the E1A mutant virus dl1505 (Osborne et al. 1984) inserted into the PstI site of pBR322. Mutant dl1505 has an out-of-frame deletion following amino acid 22 in the E1A proteins. The plasmids thus derived for the mutants are designated pBE1505/IN5, pBE1505/IN10, pBE1505/IN16, pBE1505/ IN22, pBE1505/LS - 48/ - 39, pBE1505/LS - 38/ - 31, and $\mathrm{pBE} 1505 / \mathrm{LS}-30 /-23$.

\section{Construction of E1B promoter mutant virus}

All E1B promoter mutant adenoviruses were generated by in vivo recombination after transfection of DNA into 293 cells (Stow 1981). Three micrograms of XbaI-digested dl309 (Jones and Shenk 1979b/ viral DNA and $5 \mu \mathrm{g}$ of BamHI-digested pBE1505-derived mutant plasmid DNA were cotransfected. Virus plaques isolated from the transfections were propagated, and viral DNA isolated and digested with XhoI and EcoRI to identify the desired mutants. Two further rounds of plaque formation on 293 cells were performed for each mutant virus to ensure purity.

\section{Quantitative S1 analysis of E1B RNA}

HeLa suspension cells were infected with mutant viruses at 20 plaque forming units (pfu)/cell. To analyze the transcriptional activation effect of E1A or IEP, the E1B promoter mutant viruses were coinfected with helper adenovirus S13B (20 pfu/cell) or pseudorabies virus (10 pfu/cell), respectively. S13B virus (obtained from J. Sambrook) provided functional ElA protein in trans, but its E1B gene contains a small deletion that distinguishes the S13B E1B RNA from the E1B RNA expressed from the E1B promoter mutants (Wu et al. 1987). To commence infection, absorption of adenovirus and pseudorabies virus was performed by adding virus stocks to suspension cultures of exponentially growing HeLa cells concentrated to $4 \times 10^{6}$ cells/ $\mathrm{ml}$ for $\mathrm{l} \mathrm{hr}$ and $0.5 \mathrm{hr}$, respectively. Infected HeLa cells were harvested $6 \mathrm{hr}$ postinfection, and cytoplasmic RNA was extracted as described (Berk et al. 1979).

The S1 probe was the Ad2 BstEII (nucleotide 1918) (Gingeras 
et al. 1982)-XbaI (nucleotide 1336) fragment 5' labeled with ${ }^{32} \mathrm{P}$ at the BstEII site (+220 with respect to the ElB cap site). Fifty micrograms of cytoplasmic RNA was hybridized with $75 \mathrm{ng}$ probe fragment at $46^{\circ} \mathrm{C}$ for $16-20 \mathrm{hr}$ in $50 \mu \mathrm{l}$, as described (Berk and Sharp 1977). Sl digestion was performed by adding $500 \mu \mathrm{l}$ of solution containing $0.25 \mathrm{M} \mathrm{NaCl}, 0.03 \mathrm{M} \mathrm{Na}$ acetate (pH 4.5), $1 \mathrm{mM} \mathrm{ZnCl}, 5 \%$ glycerol, and $400-500$ units of nuclease $\mathrm{S} 1$ (BRL) at $22^{\circ} \mathrm{C}$ for $60 \mathrm{~min}$. Protected probe fragments were separated by electrophoresis on a $5 \%$ denaturing polyacrylamide gel. Direct or preflashed-intensified autoradiograms were analyzed with a Hoefer densitometer, and peak integration was performed by computer (Data Acquisition Program for Acorn $\mathrm{BBCl}$.

\section{DNase I footprinting competition}

The DNase I footprint probes were prepared from clones of Ad2 wild-type or mutant sequences from 1336 (XbaI) to 1769 (SacI) into the corresponding sites of pUC18. The plasmids were digested at the EcoRI site of the pUC18 polylinker next to the SacI site $(+70)$ of the wild-type E1B promoter fragment. Phosphatase and kinase treatments followed by secondary digestion with $X b a I$ produced a probe labeled at the EcoRI site next to +70 on the transcribed strand extending to -363 . The concentrations of the competing fragments were quantitated carefully by spectrophotometry and checked by gel electrophoresis and ethidium bromide staining of aliquots.

Partially purified Spl (the $0.3 \mathrm{M} \mathrm{KCl}$ eluate from heparin agarose) was prepared according to Briggs et al. (1986) and used in the analysis of Figure. 4. Extensively purified Spl prepared by three passages over a specific-sequence DNA affinity column (Kadonaga and Tjian 1986) was used in the experiments of Figure 3. Binding reactions were carried out with $10 \mathrm{fmole}$ probe, $3.5 \mu \mathrm{g}$ of Spl fraction, and $1 \mu \mathrm{g}$ of $[$ poly $(\mathrm{dl}-\mathrm{dC})] \cdot[$ poly $(\mathrm{dl}-$ $\mathrm{dC} /(\mathrm{BRL})$ in a $50-\mu \mathrm{l}$ volume, as described (Jones et al. 1985). Titration of the Spl fraction showed that $1.4 \mu \mathrm{g}$ was sufficient to protect the probe completely. Reactions were incubated on ice for 15-20 min with or without the cold competitor fragments, followed by $2 \mathrm{~min}$ at room temperature. DNase I digestion was carried out for $1 \mathrm{~min}$ at room temperature by adding 2.5-4 ng DNase I (Worthington, freshly diluted) in $50 \mu \mathrm{l}$ of 5 $\mathrm{mM} \mathrm{CaCl} 2,10 \mathrm{~mm} \mathrm{MgCl}_{2}$. The reactions were processed further, as described (Jones et al. 1985), and resolved on an $8 \%$ polyacrylamide sequencing gel.

\section{Acknowledgments}

We are grateful to Martin Schmidt and Wes Lee for providing the Spl preparations used in the DNase I footprinting experiments and to Carol Eng for excellent technical assistance. This work was supported by grant CA25235 from the National Cancer Institute, U.S. Public Health Service. L.W. was supported by the Medical Scientist Training Program Mandel Scholarship at the University of California, Los Angeles.

\section{References}

Angel, P., M. Imagawa, R. Chiu, B. Stein, R.J. Imbra, H.J Rahmsdorf, C. Jonat, P. Herrlich, and M. Karin. 1987. Phorbol ester-inducible genes contain a common cis element recognized by a TPA-modulated trans-acting factor. Cell 49: 729-739.

Barany, F. 1985. Two-codon insertion mutagenesis of plasmid genes by using single-stranded hexameric oligonucleotides. Proc, Natl. Acad. Sci. 82: 4202-4206.
Benoist, C. and P. Chambon. 1981. In vivo sequence requirements of the SV40 early promoter region. Nature 290: 304310.

Berk, A.J. 1986. Adenovirus promoters and E1A transactivation. Annu. Rev. Genet. 20: 45-79.

Berk, A.J. and P.A. Sharp. 1977. Sizing and mapping of early adenovirus mRNAs by gel electrophoresis of S1 endonuclease-digested hybrids. Cell 12: 721-732.

Berk, A.J., F. Lee, T. Harrison, J. Williams, and P.A. Sharp. 1979. Pre-early adenovirus 5 gene product regulates synthesis of early viral messenger RNAs. Cell 17: 935-944.

Birnboim, H.C. and J. Doly. 1979. A rapid alkaline extraction procedure for screening recombinant plasmid DNA. Nucleic Acids Res. 7: 1513-1523.

Breathnatch, R. and P. Chambon. 1981. Organization and expression of eukaryotic split genes coding for proteins. Annu. Rev. Biochem. 50: 349-383.

Briggs, M.R., J.T. Kadonaga, S.P. Bell, and R. Tjian. 1986. Purification and biochemical characterization of the promoterspecific transcription factor, Spl. Science 234: 47-52.

Charnay, P., P. Mellon, and T. Maniatis 1985. Linker scanning mutagenesis of the $5^{\prime}$-flanking region of the mouse $\beta$-majorglobin gene: Sequence requirements for transcription in erythroid and nonerythroid cells. Mol. Cell. Biol. 5: 14981511.

Chodosh, L.A., R.W. Carthew, and P.A. Sharp. 1986. A single polypeptide possesses the binding and transcription activities of the adenovirus major later promoter. Mol. Cell. Biol. 6: $4723-4733$.

Coen, D.M., S.P. Weinheimer, and S.L. McKnight. 1986. A genetic approach to promoter recognition during trans induction of viral gene expression. Science 234: 53-59.

Concino, J., R.A. Goldman, M.H. Caruthers, and R. Weinmann. 1983. Point mutations in the adenovirus major late promoter with different transcriptional efficiencies in vitro. $/$. Biol. Chem. 258: 8493-8496.

Corden, J., B. Wasylyk, A. Buchwalder, P. Sassone-Corsi, C. Kedinger, and P. Chambon. 1980. Promoter sequences of eukaryotic protein-coding genes. Science 209: 1406-1413.

Dynan, W.S. and R. Tjian. 1983a. Isolation of transcription factors that discriminate between different promoters recognized by RNA polymerase II. Cell 32: 669-680.

- 1983b. The promoter-specific transcription factor Sp1 binds to upstream sequences in the SV40 early promoter. Cell 35: 79-87.

Feldman, L.T., M.F. Imperiale, and J.R. Nevins. 1982. Activation of early adenovirus transcription by the herpes-virus immediate early gene: Evidence for a common cellular control factor. Proc. Natl. Acad. Sci. 79: 4952-4956.

Fromm, M. and P. Berg. 1983. Transcription in vivo from SV40 early promoter deletion mutants without repression by large T antigen. J. Mol. Appl. Genet. 2: 127-135.

Gidoni, D., W.S. Dynan, and R. Tjian. 1984. Multiple specific contacts between a mammalian transcription factor and its cognate promoters. Nature 312: 409-413.

Gingeras, T.R., D. Sciaky, R.E. Gelinas, J. Bing-Dong, C.E. Yen, M.M. Kelley, P.A. Bullock, B.L. Parsons, K.E. O'Neill, and R.J. Roberts. 1982. Nucleotide sequences from the adenovirus-2 genome. I. Biol. Chem. 257: 13475-13491.

Graham, F., R.W. Smiley, and R. Nairn. 1977. Characteristics of a human cell line transformed by DNA from human adenovirus. J. Gen. Virol. 36: 59-72.

$\mathrm{Hu}, \mathrm{S}$. and J.L. Manley. 1981. DNA sequence required for initiation of transcription in vitro from the major late promoter of adenovirus 2. Proc. Natl. Acad. Sci. 78: 820-824.

Jones, N. and T. Shenk. 1979a. An adenovirus type 5 early gene 
function regulates expression of other early viral genes. Proc. Natl. Acad. Sci. 76: 3665-3669.

- $1979 \mathrm{~b}$. Isolation of adenovirus type 5 host range deletion mutants defective for transformation of rat embryo cells. Cell 17: 683-689.

Jones, K.A., K.R. Yamamoto, and R. Tjian. 1985. Two distinct transcription factors bind to the HSV thymidine kinase promoter in vitro. Cell 42: 559-572.

Kadonaga, J.T., K.R. Carner, F.R. Masiarz, and R. Tjian. 1987. Isolation of cDNA encoding transcription factor $\mathrm{Spl}$ and functional analysis of the DNA binding domain. Cell 51: 1079-1090.

Kadonaga, J.T., K.A. Jones, and R. Tjian. 1986. A DNA binding protein required for the promoter-specific activation of RNA polymerase II transcription in mammalian cells. Trends Biochem. Sci. 11: 20-24.

Kadonaga, J.T. and R. Tjian. 1986. Affinity purification of sequence-specific DNA binding proteins. Proc. Natl. Acad. Sci. 83: 5889-5893.

Lee, W., P. Mitchell, and R. Tiian. 1987a. Purified transcription factor AP-1 interacts with TPA-inducible enhancer elements. Cell 49: 741-752.

Lee, W., A. Haslinger, M. Karin, and R. Tjian. 1987b. Activation of transcription by two factors that bind promoter and enhancer sequences of the human metallothionein gene and SV40. Nature 325: 368-372.

Leong, K., L. Brunet, and A.J. Berk. 1988. Factors responsible for the higher transcription activity of adenovirus infected cell extracts fractionate with the TATA-box transcription factor. Mol. Cell. Biol. (in press).

Maniatis, T., S. Goodbourn, and J.A. Fischer. 1987. Regulation of inducible and tissue-specific gene expression. Science 236: $1237-1244$.

McKnight, S.L. 1982. Functional relationships between transcriptional control signals of the thymidine kinase gene of herpes simplex virus. Cell 31: 355-365.

McKnight, S.L. and R. Kingsbury. 1982. Transcriptional control signals of a eukaryotic protein coding gene. Science 217: 316-324.

McKnight, S. and R. Tjian. 1986. Transcriptional selectivity of viral genes in mammalian cells. Cell 46: 795-805.

Mitchell, P.J., C. Wang, and R. Tjian 1987. Positive and negative regulation of transcription in vitro: Enhancer-binding protein AP-2 is inhibited by SV40 T antigen. Cell 50: 847861.

Nevins, J.R. 1981. Mechanism of activation of early viral transcription by the adenovirus E1A gene product. Cell 26: 213220.

Osborne, T.F., D.N. Arvidson, E.S. Tyau, M. DunsworthBrowne, and A.J. Berk. 1984. Transcription control region within the protein-coding portion of adenovirus E1A genes. Mol. Cell. Biol. 4: 1293-1305.

Parker, C.S. and J. Topol. 1984. A Drosophila RNA polymerase II transcription factor contains a promoter-region-specific DNA-binding activity. Cell 36: 357-369.

Ptashne, A. 1986. Gene regulation by proteins acting nearby and at a distance. Nature 322: 697-701.

Sanger, F., S. Nicklen, and A.R. Coulson. 1977. DNA sequencing with chain terminating inhibitors. Proc. Natl. Acad. Sci. 74: 5463-5467.

Sawadogo, M. and R.G. Roeder. 1985. Interaction of a gene-specific transcription factor with the adenovirus major late promoter upstream of the TATA-box region. Cell 43: 165-175.

Smith, A.J.H. 1980. DNA sequence analysis by primed synthesis. Methods Enzymol. 65: 560-580.

Stow, N.D. 1981. Cloning of a DNA fragment from the left hand terminus of the adenovirus type 2 genome and its use in site-directed mutagenesis. J. Virol. 37: 171-180.

Takahashi, K., M. Vigneron, H. Matthes, A. Wildeman, M. Zenke, and P. Chambon. 1986. Requirement of stereospecific alignments for initiation from the simian virus 40 early promoter. Nature 319: 121-126.

Wasylyk, B., C. Wasylyk, H. Matthes, M. Wintzerith, and P. Chambon. 1983. Transcription from the SV40 early-early and late-early overlapping promoters in the absence of DNA replication. EMBO /. 2: 1605-1611.

Wildeman, A.G., M. Zenke, C. Schatz, M. Bintzerith, T. Grunstrom, H. Matthes, K. Takahashi, and P. Chambon. 1986 Specific protein binding to the simian virus 40 enhancer in vitro. Mol. Cell. Biol. 6: 2098-2105.

Wu, L., D.S.E. Rosser, M.C. Schmidt, and A. Berk. 1987. A TATA box implicated in E1A transcriptional activation of a simple adenovirus 2 promoter. Nature 326: $512-515$.

Zoller, M.J. and M. Smith. 1983. Oligonucleotide-directed mutagenesis of DNA fragments cloned into M13 derived vectors. Methods Enzymol. 100: 468-501. 


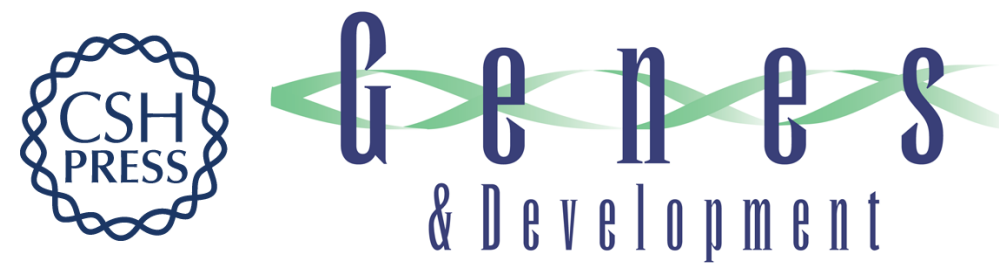

\section{Constraints on spacing between transcription factor binding sites in a simple adenovirus promoter.}

$\mathrm{L}$ Wu and A Berk

Genes Dev. 1988, 2:

Access the most recent version at doi:10.1101/gad.2.4.403

References This article cites 48 articles, 18 of which can be accessed free at:

http://genesdev.cshlp.org/content/2/4/403.full.html\#ref-list-1

License

Email Alerting

Service

Receive free email alerts when new articles cite this article - sign up in the box at the top right corner of the article or click here.

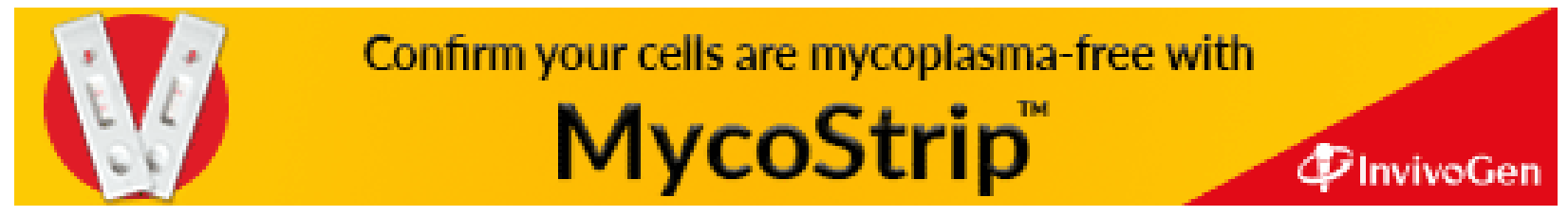

